\title{
INTERAÇÃO CALAGEM-ADUBAÇÃO NITROGENADA NA PRODUÇÃO DE SORGO SOB DEFICIÊNCIA HÍDRICA EM ROTAÇÃO COM SOJA (')
}

\author{
PAULO BOLLER GALLO $(2,5)$, \\ HIPÓLITO ASSUNÇÃO ANTONIO MASCARENHAS $(3,5)$, \\ ONDINO CLEANTE BATAGLIA $(4,5)$ e JOSÉ ANTONIO QUAGGIO $(4,5)$
}

\begin{abstract}
RESUMO
No ano agrícola 1983/84, conduziu-se um exerimento num Latossolo Vermelho-Escuro álico na Estação Experimental de Mococa, SP, plantando o sorgo cultivar Contigrão $111 \mathrm{em}$ parcelas que receberam calagem em 1980 nas doses equivalentes a 1, 4, 7 e 10t/ha de calcário dolomítico, e foram cultivadas por três anos com soja. Nas subparcelas, aplicaram-se 0,40 , 80 e $120 \mathrm{~kg} / \mathrm{ha}$ de nitrogênio em cobertura 35 dias depois da germinação. Os resultados mostraram que a calagem elevou substancialmente a produção, mesmo com a drástica deficiência hídrica ocorrida na fase reprodutiva da cultura. A calagem eliminou as limitaçōes impostas pela toxicidade de alumínio e aumentou a disponibilidade do nitrogênio proveniente dos restos da cultura de soja. Em todos os níveis de calagem, houve aumento linear de concentração de nitrogênio nas folhas de sorgo, em função das doses de nitrogênio em cobertura, mas as quantidades necessárias para atingir níveis adequados de $\mathrm{N}$ nas folhas foram substancialmente reduzidas nos níveis mais altos de calagem.
\end{abstract}

Termos de indexação: soja, sorgo, adubação nitrogenada, toxicidade de Al, calagem.

(1) Trabalho apresentado na XVII Reunião Brasileira de Fertilidade do Solo, Londrina (PR), 27 de julho-1 ${ }^{\circ}$ de agosto de 1986. Recebido para publicaçāo em 10 de setembro de 1985. pinas (SP).

(2) Estação Experimental de Mococa, Instituto Agronômico (IAC), Caixa Postal 28, 13001 Cam-

(3) Seção de Leguminosas, IAC.

( $\left.{ }^{4}\right)$ Seção de Fertilidade do Solo e Nutrição de Plantas, IAC.

(5) Com bolsa de suplementação do CNPq. 


\section{INTRODUÇÃO}

O sorgo é uma planta bastante atraente como opção de plantio no período subseqüente a uma cultura de verāo de ciclo curto, por sua relativa tolerância às condições de limitação hídrica, podendo ser também uma alternativa viável como cultura de verão. Todavia, para desenvolver todo seu potencial de exploração de água e nutrientes, precisa de condiçōes de solo favoráveis ao desenvolvimento radicular, sobretudo em relação à acidez do solo, por se tratar de uma espécie relativamente sensivel à toxicidade de alumínio, embora existam diferenças marcantes entre cultivares (FURLANI et alii, 1984).

Gramineas cultivadas após soja têm-se beneficiado dos resíduos dessa cultura especialmente em relação ao aproveitamento do nitrogênio residual, como demonstram trabalhos efetuados com milho (DERPSCH et alii, 1985, GALLO et alii, 1983, e MASCARENHAS et alii, 1978), trigo (BATAGLIA et alii, 1983, e OLIVEIRA et alii, 1979) e arroz-de-sequeiro (PEREIRA et alii, 1979).

A calagem, por outro lado, proporciona maior desenvolvimento à cultura de soja e, conseqüentemente, maior produção de nitrogênio residual (QUAGGIO et alii, 1982). Assim, é possivel que o cultivo de gramíneas depois de soja, em solo cuja acidez tenha sido corrigido pela calagem, apresente respostas menos acentuadas à adubação nitrogenada. O ọjetivo deste trabalho foi testar essa hipótese, utilizando-se a cultura do sorgo-granifero.

\section{MATERIAL E MÉTODOS}

No presente experimento, instalado no ano agrícola de 1983/84, em Latossolo Vermelho-Escuro álico, na Estação Experimental de Mococa, aproveitou-se um ensaio permanente de calagem iniciado em 1973, num delineamento experimental em blocos casualizados, com quatro repetições, em esquema de parcelas subdivididas. Nas parcelas, aplicou-se calcário nas doses $0,3,6$ e 9t/ha, apenas em 1973 e, nas subparcelas, anualmente, doses de nitrogênio e fósforo, num fatorial nitrogênio $x$ fósforo para o milho, cujos detalhes experimentais foram descritos por CAMARGO et alii (1982); esse experimento foj conduzido durante cinco cultivos sucessivos de milho, seguidos por algodão e soja.

As análises do solo antes da aplicação de calagem em 1980 acham-se no quadro 1. As doses utilizadas foram: 1, 4, 7 e 10t/ha respectivamente. Em seguida, plantou-se soja por três anos consecutivos com a adubação anual de $0-60-0 \mathrm{~kg} / \mathrm{ha}$ de $\mathrm{N}-\mathrm{P}_{2} \mathrm{O}_{5}-\mathrm{K}_{2} \mathrm{O}$ respectivamente. $\mathrm{Na}$ época de colheita, foi retirada a parte aérea de cada parcela, deixando-se apenas as raízes, que foram incorporadas com a preparação subseqüente do solo. 
QUADRO 1. Análise do solo antes da aplicação de calagem em 1980, nos tratamentos que receberam calagem em 1973

\begin{tabular}{|c|c|c|c|c|c|c|c|}
\hline $\begin{array}{l}\text { Calagem } \\
\text { em } 1973\end{array}$ & MO & $\mathrm{pH}$ & $\mathrm{Al}$ & $\mathrm{Ca}$ & $\mathrm{Mg}$ & $\mathrm{K}$ & $\mathbf{P}$ \\
\hline t/ha & $\%$ & & 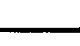 & $\mathrm{meq} / \mathrm{cm}^{2}$ solo & & & $\mu \mathrm{g} / \mathrm{ml}$ \\
\hline $\mathbf{0}$ & 2,77 & 4,49 & 1,30 & 0,31 & 0,11 & 0,16 & 14 \\
\hline 3 & 2,86 & 4,81 & 0,82 & 0,78 & 0,28 & 0,21 & 12 \\
\hline 6 & 2,92 & 5,09 & 0,40 & 1,25 & 0,51 & 0,25 & 12 \\
\hline 9 & 2,76 & 5,30 & 0,28 & 1,63 & 0,74 & 0,25 & 11 \\
\hline
\end{tabular}

No quarto ano, plantou-se sorgo, cultivar Contigrão 111, mantendo-se o delineamento inicial em parcelas subdivididas, aplicando-se, porém, nas subparcelas, as doses de $0,40,80$ e $120 \mathrm{~kg} / \mathrm{ha}$ de nitrogênio em cobertura, na forma de sulfato de amônio, 35 dias após a germinação.

Cada subparcela, com $4 \times 10 \mathrm{~m}$, possuía cinco linhas de sorgo espaçadas de $0,80 \mathrm{~m}$, utilizando-se para a avaliação da produção e amostragens de solos e folhas apenas as três linhas centrais. Todas essas parcelas receberam como adubação básica, no sulco de plantio, $16,56,32$ e 0,8kg/ha de $\mathrm{N}, \mathrm{P}_{2} \mathrm{O}_{5}$, $\mathrm{K}_{2} \mathrm{O}$ e $\mathrm{Zn}$ respectivamente, através da fórmula 4-14-8 + 0,2\% de zinco.

As amostras para análise foliar foram colhidas no inicio de formação das panículas, coletando-se as folhas +3 ou +4 . As análises de macro e micronutrientes nas folhas foram feitas de acordo com os métodos de BATAGLIA et alii (1983).

Foram feitas amostragens do solo nas profundidades de $0-20 \mathrm{~cm}$ e $20-40 \mathrm{~cm}$, sendo as análises químicas do solo efetuadas pelos métodos descritos por RAIJ \& QUAGGIO (1983).

\section{RESULTADOS E DISCUSSÃO}

Durante a fase inicial da cultura, a quantidade e a distribuição de chuvas proporcionaram excelente desenvolvimento vegetativo das plantas, especialmente nos tratamentos com as doses mais elevadas de nitrogênio em cobertura e de calcário. Entretanto, a partir do inicio do florescimento até a colheita, um periodo de deficiência hídrica muito intenso, de certa forma, reduziu a produtividade do sorgo no experimento (Figura 1). 


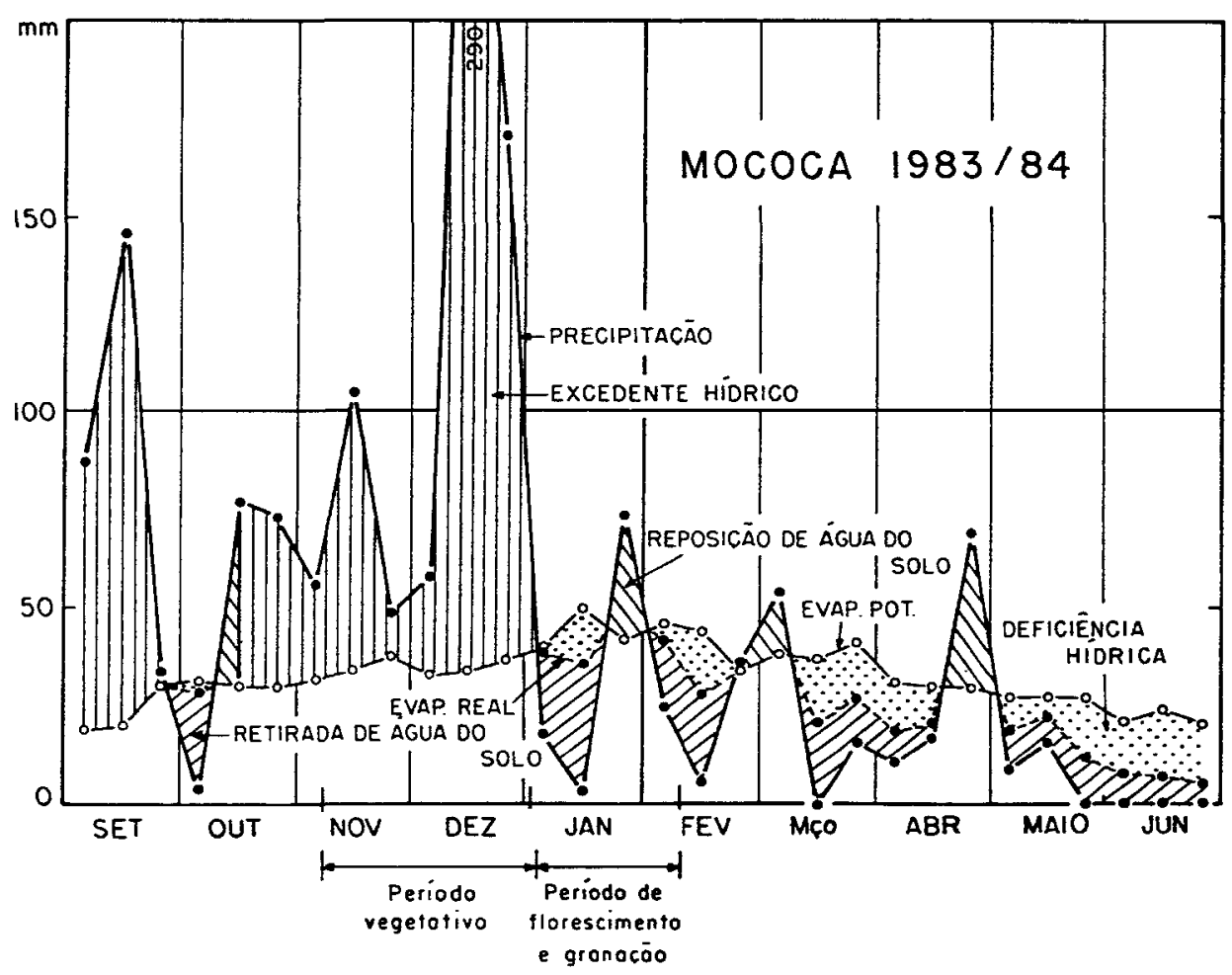

FIGURA 1. Balanço hídrico pela metodologia de Thornthwaite no ano agrícola de 1983/84, na Estação Experimental de Mococa, SP.

$\mathrm{Na}$ figura 2, verifica-se que houve considerável aumento de produção em função dos niveis de calagem, independente das doses de nitrogênio aplicadas em cobertura. Esse aumento está provavelmente associado com a elevação no indice de saturação em bases no solo, que cresceu de 23 até $67 \%$ na camada $0-20 \mathrm{~cm}$, com um conseqüente decréscimo nas concentrações de aluminio nas folhas de 424 para 164 ppm (Quadro 2). Verifica-se, ainda, que, apesar das doses elevadas de calcário, houve pequena correção de acidez na camada 20-40 cm; nela, a saturação de Al foi bastante elevada nos tratamentos com as doses mais baixas de calcário. Por essa razão, as observaçōes de campo mostraram que as raízes estavam bastante concentradas na camada superficial do solo. Nas doses mais elevadas de calcário, houve melhor correção da acidez em subsuperfície, o que possibilitou maior aprofundamento do sistema radicular, reduzindo-se, portanto, os efeitos do déficit hídrico sobre a produtividade do sorgo. 


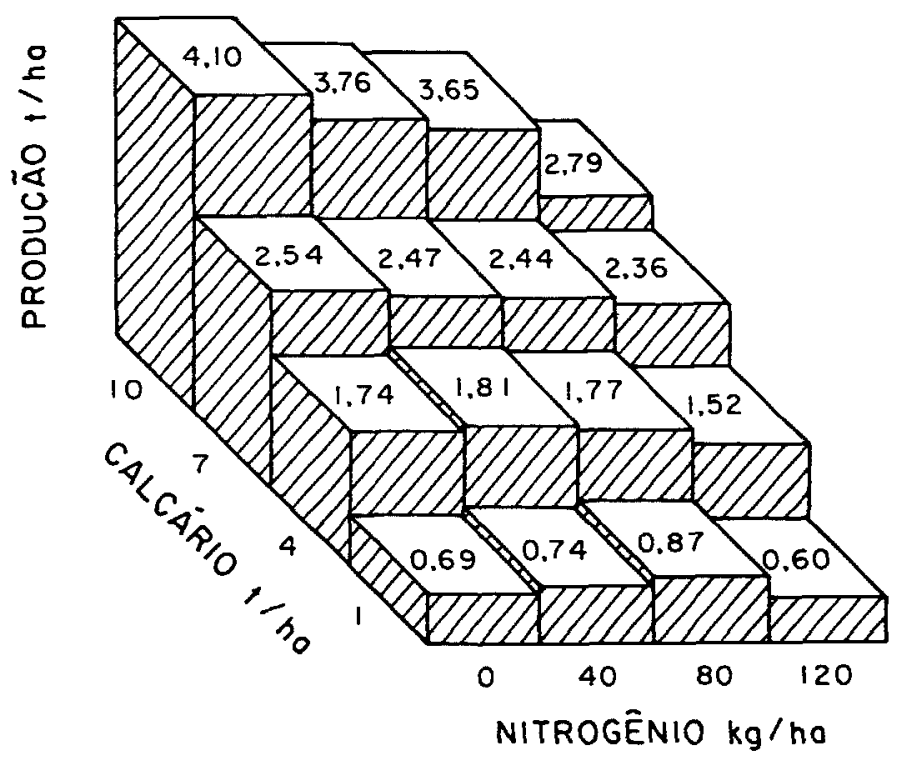

FIGURA 2. Produção média de grão de sorgo (t/ha) em função de doses de calcário e de nitrogênio em cobertura.

QUADRO 2. Influência da aplicação de doses de calcário sobre a concentraçāo de macro e micronutrientes e alumínio nas folhas de sorgo e nos valores de saturação por bases e alumínio em duas profundidades no solo

\begin{tabular}{|c|c|c|c|c|c|c|c|c|c|}
\hline \multirow{2}{*}{$\begin{array}{l}\text { Calcário } \\
\text { aplicado }\end{array}$} & \multirow{2}{*}{$\mathbf{K}$} & \multirow{2}{*}{$\mathrm{Ca}$} & \multirow{2}{*}{$\mathbf{M g}$} & \multirow{2}{*}{$\mathrm{Mn}$} & \multirow{2}{*}{$\mathrm{Al}$} & \multicolumn{2}{|c|}{ Satur. bases no solo } & \multicolumn{2}{|c|}{ Satur. Al no solo } \\
\hline & & & & & & $0-20 \mathrm{~cm}$ & $20-40 \mathrm{~cm}$ & $0-20 \mathrm{~cm}$ & $20-40 \mathrm{~cm}$ \\
\hline tha & 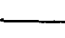 & $\%$ & - & -1 & $\ldots$ & - & {[} & & \\
\hline 1 & 1,33 & 0,46 & 0,19 & 65,6 & 424 & 23 & 10 & 59 & 71 \\
\hline 4 & 1,43 & 0,47 & 0,28 & 62,8 & 300 & 37 & 15 & 33 & 61 \\
\hline 7 & 1,32 & 0,50 & 0,36 & 49,8 & 224 & 54 & 21 & 0 & 55 \\
\hline 10 & 1,37 & 0,48 & 0,40 & 43,7 & 164 & 67 & 31 & $\mathbf{0}$ & 36 \\
\hline
\end{tabular}

Se, por um lado, houve efeito marcante da calagem sobre a produtividade, por outro, dentro de cada nivel de calagem, não houve resposta significativa ao nitrogênio, chegando inclusive a ter efeito depressivo, principalmente na dose mais elevada de calcário (Figura 3). Esse resultado, que não era de esperar, certamente ocorreu em função das condições climáticas que prevaleceram durante o ciclo da cultura. Nas parcelas com 10t/ha de calcário, observou-se exuberante desenvolvimento vegetativo e, à medida que as doses de nitrogênio aumentaram, a maior área foliar resultante e, possivelmente, a maior capacidade de ajusta- 
mento osmótico nas plantas bem supridas de nitrogênio (BATAGLIA et alii, 1985), associadas a um sistema radicular desenvolvido, devem ter contribuido para uma exaustão rápida da água disponível no solo durante a fase reprodutiva da cultura. Além disso, em vista da seca e do crescimento vegetativo, houve acamamento de muitas plantas, à medida que se aumentaram as doses de nitrogênio aplicado.

$\mathrm{Na}$ figura 3, observa-se que as concentrações de nitrogênio nas folhas aumentaram linearmente em função da adubação, independente das doses de calcário. Nota-se, entretanto, que na dose zero de nitrogênio em cobertura houve considerável aumento na concentração de nitrogênio nas folhas, devido à calagem, provavelmente em função não só do maior desenvolvimento radicular, mas, sobretudo, da maior disponibilidade do nutriente nos restos de cultura da soja anteriormente cultivada por três anos nos mesmos canteiros. Considerando-se que, na ausência de outras limitaçōes, o nivel de $3 \%$ de nitrogênio nas folhas seja adequado para se atingirem boas produçōes, segundo JONES \& ECK (1973) e HIROCE et alii (1981), observa-se que, para atingir esse nível, foram necessários cerca de $100 \mathrm{~kg} / \mathrm{ha}$ de nitrogênio na dose de $1 \mathrm{t} / \mathrm{ha}$ e menos de $30 \mathrm{~kg} / \mathrm{ha}$ de nitrogênio para as outras duas doses mais elevadas. Esses indices dāo uma idéia da economia que pode ser conseguida utilizando-se a rotação de cultura para aproveitar os residuos da soja como fonte de nitrogênio para o sorgo, principalmente se ele for plantado como segunda cultura, logo após a colheita da soja, conforme vem sendo praticado em larga escala na região da Alta Mojiana no Estado de Sāo Paulo.

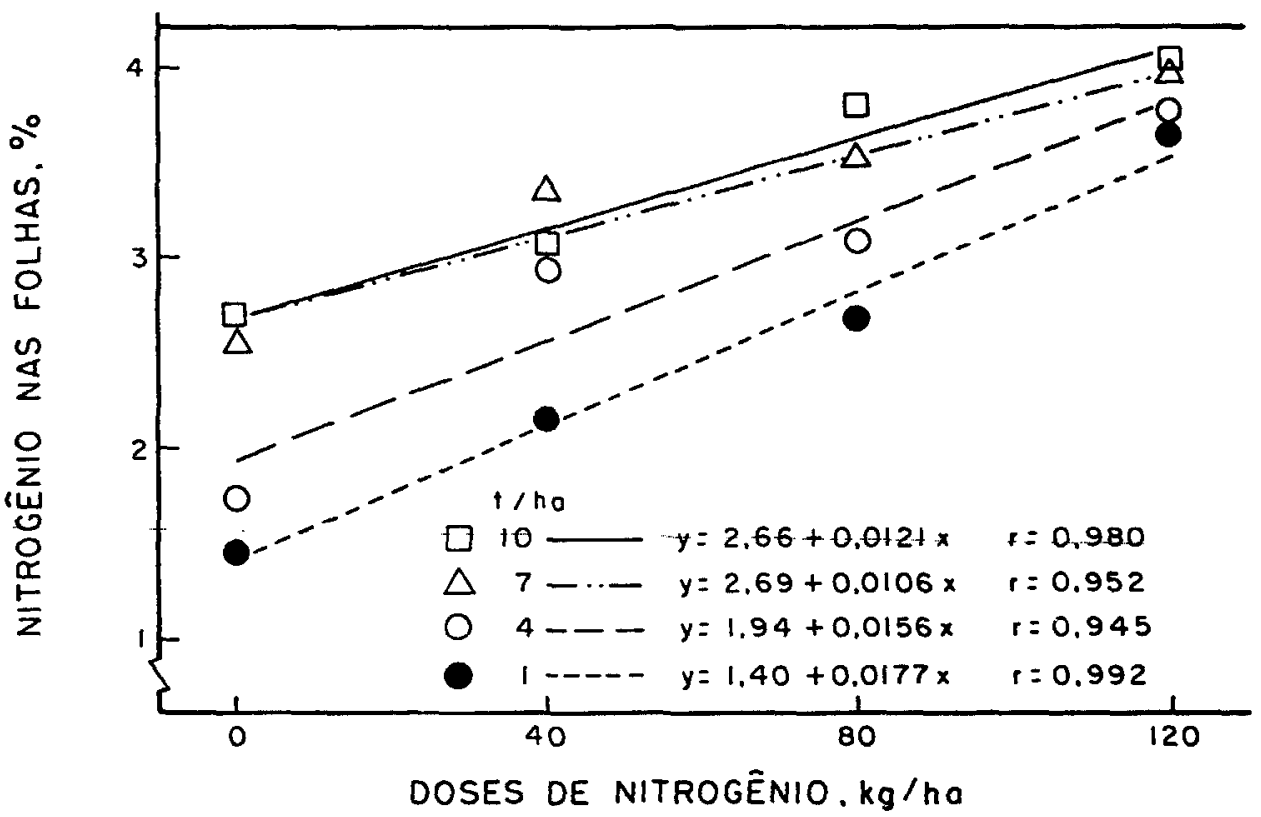

FIGURA 3. Efeito da adubação nitrogenada sobre o teor de $\mathrm{N}$ em folhas de sorgo 


\section{SUMMARY}

\section{INTERATION OF LIME AND NITROGEN FERTILIZATION ON THE YIELD OF SORGHUM UNDER DROUGHT IN ROTATION WITH SOYBEANS}

An experiment was conducted during the summer of 1983/84 in a clay textured allic, dark red Latosol at Mococa Experimental Station, State of São Paulo, Brazil. Sorghum Contigrão 111 was planted in plots that had received the equivalent to $1,4,7$ and 10 t/ha of dolomitic limestone in 1980 . These plots were cultivated continuously with soybeans during the three previous years. In the subplots, $0,40,80$ and 120 of $\mathrm{N}$ were applied as side dressing 35 days after germination. The results showed that liming increased the yield of grain sorghum despite the drought at the seed filling stage. It also decreased the toxic effect of aluminum and increased the availability of $N$ from the residues of previous soybean crops. At all the liming rates a linear increase $w$ as observed in the leaf $\mathbf{N}$ concentration as a function of the rates of nitrogen applied as side dressing. The quantity of $\mathrm{N}$ fertilizer required to attain adequate leaf $\mathrm{N}$ concentration was substancially reduced at the higher rates of lime applied.

Index terms: soybeans, sorghum, nitrogen fertilization, Al toxicity, liming.

\section{REFERÊNCIAS BIBLIOGRÁFICAS}

BATAGLIA, O.C.; QUAGGIO, J.A.; BRUNINI, O. \& CIARELLI, D.M. Adubação nitrogenada e ajustamento osmótico em milho e sorgo. Pesquisa Agropecuária Brasileira, Brasilia, 20(6):659-665, 1985.

; TEIXEIRA, J.P.F.; FURLANI, P.R.; FURLANI, A.M.C. \& GALLO, J.R. Métodos de análise química de plantas. Campinas, Instituto Agronômico, 1983. 48p. (Boletim, 78)

CAMARGO, A.P. de; RAIJ, B. van; CANTARELLA, H.; ROCHA, T.R. da; NAGAI, V. \& MASCARENHAS, H.A.A. Efeito da calagem nas produçōes de cinco cultivos de miIho, seguidos de algodão e soja. Pesquisa Agropecuária Brasileira, Brasília, 17:1007$-1112,1982$.

DERPSCH, R.; SIDIRAS, N. \& HEINZMANN, F.X. Manejo do solo com coberturas verdes de inverno. Pesquisa Agropecuária Brasileira, Brasília, 20(7):761-773, 1985.

FURLANI, P.R.; BASTOS, C.R.; BORGONOVI, P.A. \& SCHAFFEPT, R.E. Resposta diferencial de genótipos de sorgo para tolerância ao alumínio em solução nutritiva. In: CONGRESSO NACIONAL DE MILHO E SORGO, 15., Macei6, 1984. Resumos. p.41.

GALLO, P.B.; LAVORENTI, A.; SAWAZAKI, E.; HIROCE, R. \& MASCARENHAS, H.A.A. Efeito de culturas anteriores de soja na produção e no teor de nitrogênio das folhas e dos grāos de milho. Revista Brasileira de Ciência do Solo, Campinas, 7:149152, 1983.

HIROCE, R.; SAWAZAKI, E.; POMMER, C.V. \& MIRANDA, L.T. de. Efeitos da adubação NPK na produção e na composição mineral de folhas de diferentes cultivares de sorgo e milho. Revista Brasileira de Ciência do Solo, Campinas, 5:67-71, 1981. 
JONES, J.B. \& ECK, H.V. Plant analysis as an aid in fertilizing corn and grain sorghum. In: WALSH, L.M. \& BEATON, J.D. Soil testing and plant analysis. Madison, Soil Science Society of America, 1973. p.349-364.

MASCARENHAS, H.A.A.; HIROCE, R.; BRAGA, N.R.; MIRANDA, M.A.C. de; POMMER, C.V. \& SAWAZAKI, E. Efeito de nitrogênio residual de soja na produção de milho. Campinas, Instituto Agronômico, 1978. 16p. (Boletim técnico, 58)

OLIVEIRA, O.F.; FELÍCIO, J.C.; MASCARENHAS, H.A.A. \& HIROCE, R. Efeito do nitrogênio residual de soja na produção de trigo. Bragantia, Campinas, 38:LIII-LVI, 1979. (Nota, 13)

PEREIRA, J.C.V.N.A.; MASCARENHAS, H.A.A.; HIROCE, R. \& CAMARGO, R. Efeito de nitrogênio e da rotação com soja na produção de arroz de sequeiro. Bragantia, Campinas, 38:LVII-LIX, 1979. (Nota, 12)

QUAGGIO, J.A.; MASCARENHAS, H.A.A. \& BATAGLIA, O.C. Resposta da soja à aplicação de doses crescentes de calcário em Latossolo Roxo distrófico de cerrado. II. Efeito residual. Revista Brasileira de Ciência do Solo, Campinas, 6:113-188, 1982.

RAIJ, B. van \& QUAGGIO, J.A. Método de análise de solo para fins de fertilidade. Campinas, Instituto Agronômico, 1983. 31p. (Boletim técnico, 81) 